\title{
Changing Paradigms: From Schooling to Schools as Adaptive Recommendation Systems
}

\author{
Anne Kristine Petersen, Rene B Christiansen*, Karsten Gynther \\ Center for Teaching and Learning, University College Absalon, Roskilde, Denmark
}

Copyright $\subset 2017$ by authors, all rights reserved. Authors agree that this article remains permanently open access under the terms of the Creative Commons Attribution License 4.0 International License

\begin{abstract}
The paper explores a shift in education from educational systems requiring student adaptation to educational recommendation systems adapting to students' individual needs. The paper discusses the concept of adaptation as addressed in educational research and draws on the system theory of Heinz von Foerster to shed light on how the educational system has used and understood adaptation. In this context, we point out two different approaches to educational adaptation: 1) students adapting to the educational system and 2) the attempt of the educational system to adapt to students through automatized system adaptation and recommendation systems. These different understandings constitute a design framework that is used to analyze two current trends: Adaptive learning systems and learning analytics. Finally, the paper discusses the potential of looking at adaptation as recommendation systems by presenting a case, which is methodologically inspired by Design-Based Research, in the form of a special type of adaptive MOOC, the so-called adaptive SPOC (Small Private Online Course).
\end{abstract}

Keywords Adaptation, Adaptive Learning Systems, MOOC, SPOC, DBR, Educational Design Research, Learning Analytics, Cybernetics, Non-trivial Machines

\section{Introduction}

Adaptation is not a new phenomenon in education; good teachers have always adapted to their students' individual needs by, for instance, modifying the way content is presented, the academic level, the sequencing of assignments or tasks, and the nature of the guidance and feedback provided. However, with the advancement of what Siemens et al. [1] refer to as fifth-generation educational technologies, adaptive learning has reached a new momentum within the field of educational research, and teachers and students are facing new opportunities and challenges as adaptive learning systems are implemented throughout the educational sphere.
In this paper, we will explore two fundamentally different approaches to educational adaptation, but to understand the challenges that we are currently facing, we must first look at the historical and social context of the concept.

Adaptation can be broadly defined as "the capability exhibited by an organic or an artificial organism to alter its behavior according to the environment" [2]. The concept of adaptation has been discussed within the field of evolutionary science for centuries, and two opposing approaches have gained ground. According to Charles Darwin and Herbert Spencer, the survival of the fittest depends on the ability of the individual to adapt to its environment; those who have the greatest adjustive capacity, are those who succeed [3, 4]. Following this line of argument, institutions have come to serve as instruments of natural selection by demanding adaptation from those entering the system. In this tradition, adaptation within an educational context becomes a deterministic means to anticipate natural selection; by predicting who will be able to succeed within the existing structures of the educational system, failure can be reduced and challenges, which might under other circumstances have forced the system to change, are eliminated [5].

Experimental scientists, on the other hand, explain evolution along the lines of James and Dewey, arguing that a human being's chances of success rely on his intelligence; humans must change the environment to their needs rather than simply accepting the environing world as it is. In this tradition, humans are seen as essentially independent and creative, and attempts to predict a person's behavior are regarded as pointless [5]. In an educational context, this approach is consequently concerned with finding the one best way to reach the highest average in terms of student performance.

This leaves us with two antagonistic approaches; one which accepts the institution and seeks out those who fit into its existing structures, and one which challenges any institution that does not comply with a generalized idea of the intelligent human being. According to Cronbach, however, both approaches result in limited social benefit, 
because they deal with adaptation on a merely abstract level without acknowledging that adaptation is always context-specific. Discussing adaptation in relation to applied psychology, Cronbach argues that "if for each environment there is a best organism, for every organism there is a best environment" [5] and for this reason, the goal of applied psychology should be to "find for each individual the treatment to which he can most easily adapt". In line with this, we will put forward the argument that in education, adaptation should be about shaping for each student the best learning environment by recommending a unique learning path that takes into consideration the individual needs of the student.

Ideally, a technology enhanced, adaptive learning system does what the expert teacher does, but in addition to this, it allows for scaling and hence improves the effectiveness and efficiency of educational adaptation. The ultimate goal of an adaptive learning system is to personalize teaching and learning in order to accelerate the student's learning outcome. This goal can be obtained if the adaptive learning system is able to identify what a student does not know or is unable to do, identify and recommend content that will allow the student to learn it, and assess the student's performance until $\mathrm{s} / \mathrm{he}$ has achieved a specific learning outcome. At a practical level, adaptive learning systems thus address a number of challenges that have always dogged educators: Students in the same class have different academic levels and bring with them different types of knowledge and skills; the content provided is either too easy or too difficult, which tends to frustrate students; and finally, teachers find it hard to adapt to students' individual needs because one-to-one instruction remains a utopian dream due to the harsh economic realities of most educational institutions [6].

Even though adaptive learning systems share the same overall goals of improving the student's individual learning outcome, such systems can take many different forms once implemented. In this paper, we will discuss two fundamentally different approaches to adaptation in education: The first approach is based on the underlying assumption that the student must adapt to the educational system, whereas the second approach requires the educational system to adapt to the student. To illustrate the latter approach, we will discuss two different kinds of adaptation modes in which the system adapts to the student, namely automatized system adaptation and recommendation systems. Finally, we will turn our attention to the potentials of adaptive recommendation systems by presenting a case in the form of an adaptive SPOC (Small Private Online Course). A SPOC is a crossing between a MOOC (Massive Open Online Course) and an online course that is small rather than massive and private rather than open [7].

\section{A Brief History of MOOCs}

The concept of MOOCs is a relatively new phenomenon in the history of distance learning. In 2013, The Horizon Report identified the development of MOOCs as "the most important trend in education" [8]. MOOCs are new forms of distance learning, and we are yet to find out whether they are "expanded forms of online higher education", as proposed by Evans \& Myrick [9], or the newest fad in online distance learning that will soon be written off in favor of the next educational quick fix. The MOOC concept originated in 2008 when the first MOOC was introduced, and various MOOC designs have since then evolved from two fundamentally different types of MOOCs: The so-called cMOOC and the xMOOC. The latter is influenced by traditional e-learning courses in distance learning, while the first was born from the theory of connectivism [10] and emphasizes collaboration and the production of text, video or artefacts in addition to bringing learners together. In 2012, Siemens stated that the difference between the two types of MOOCs is that a "cMOOC model emphasizes creation, creativity, autonomy and social networking learning" and focuses "on knowledge creation and generation", whereas the $\mathrm{xMOOC}$ model emphasizes "a more traditional learning approach through video presentations and short quizzes and testing and focuses on knowledge duplication" [11].

In the last five to six years, however, MOOC designs have drawn inspiration from both approaches, and more "blended" formats have appeared that combine elements from both camps: "What we are starting to see now, is a move away from the cMOOC/xMOOC binary toward recognition of the multiplicity of MOOC designs, purposes, topics and teaching styles" [12]. The MOOC milieu, from which our SPOC derives, is a blended MOOC that draws on principles from both the cMOOC and xMOOC models as well as other models. The emergence of blended formats highlights the need for a more balanced understanding of how MOOC designers subscribe to various ideas of learning, teaching, participation, content production and collaboration.

\section{Design Framework and Classification of the Concept of Adaption}

This section aims at discussing different understandings of the concept of adaptation within the educational system. We identify two fundamentally different approaches to educational adaptation: 1) students adapting to the educational system and 2) the attempt of the educational system to adapt to the students. Furthermore, we divide the latter into two kinds of adaptation modes: 2a) automatized system adaptation and $2 \mathrm{~b}$ ) recommendation systems. We then move on to discuss two examples of current trends; adaptive learning systems and learning analytics. But first we begin our journey in second-order cybernetics using the machine as a metaphor for understanding the relationship between the educational system and the students.

The Austrian physicist and founding father of second-order cybernetics and early constructivist learning 
theory, Heinz von Foerster, has, inspired by the work of Alan Turing, proposed the machine as a metaphor. His distinction between trivial and non-trivial machines, which originally set out to say something about cognitive behavior, can, as von Foerster later did himself, be used to criticize the educational system and the concept of adaptation. Some researchers, among them von Foerster himself, have argued that the distinction between trivial and non-trivial has proved to be an "ideales Instrument und Argument für das Aufzeigen eines Paradigmenwechsels für die Schule" [13]. Von Foerster labelled the school and the educational system "Eine Anstalt zur Trivialisierung von Menchen", i.e. a factory with the aim of making people trivial, referring to the pupil-teacher relationship as a trivial relationship or a trivial-machine: If you insert a certain input you will get an output that is known and recognized by the system beforehand. In relation to this, von Foerster criticizes rote learning and the overall focus on Memorization in schools. The processes taking place are analytically determinable and therefore predictable. Graphically von Foerster has presented the trivial-machine in the following way:

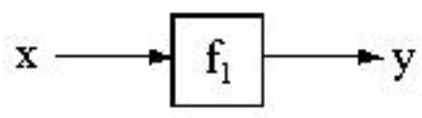

Figure 1. The trivial-machine [14]

The $\mathrm{x}$ in Fig. 1 specifies a certain input, which might, for example, be a question posed by a teacher to a pupil (f1). The output $\mathrm{y}$ is the response from the pupil to the specific question or input, which is known beforehand by the system/teacher.

The basic problem is, says von Foerster, that people are not trivial-machines. You cannot assume a certain output based on a specific input. For this reason, he proposes the concept of a non-trivial-machine: You can insert a certain input, but the output is never known beforehand. The non-trivial machine is analytically un-determinable and unpredictable. The non-trivial machine can be illustrated as follows where the $\mathrm{S}$ indicates a stimulus, which Von Foerster labels "an internal logic where the operator changes with every operation:

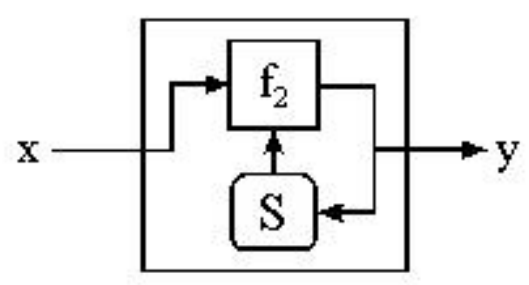

Figure 2. The Non-trivial machine [14]

How is this basic discussion related to the concept of adaptation? We propose that the metaphors proposed by von Foerster can be used to unmask a certain understanding of adaptation in educational design, namely automatized system adaptation, which is often put forward as learner (and teacher) empowerment, because educational designs using automatized system adaptation change according to student behavior. We use the term unmask because this is done on the premises of the system itself, not the learners, and in terms of von Foerster's metaphor, automatized system adaptation is a trivial position.

In a traditional educational context, students have always been forced to adapt to the system/school; students are obliged to learn to behave in a certain way, they have to show up on specific dates and at specific times, and they have to learn a specific language $[15,16]$. The underlying assumption is that if you put in a pupil, i.e. an input, you will get a certain output, i.e. a specially designed student who has learned to adapt to the system. This is masterfully portrayed in Pink Floyd's "Another Brick in the Wall Part 2" with the famous children's chorus: "We don't need no education, we don't need no thought control", but in schools, as Philip W. Jackson concluded in 1968, "all is lost, when group control is lost" [16]. This position has met substantial critique for not teaching for human beings but teaching for the system. Situated learning [17], student-based teaching [18] and teaching and learning for essential competencies such as the 21st Century Learning Skills [19] are among the many strategies to fight the critique of the educational system: "Too often the practices of contemporary schooling deny students the chance to engage the relevant domain culture, because that culture is not in evidence. Although students are shown the tools of many academic cultures in the course of a school career, the pervasive cultures that they observe, in which they participate, and which some enter quite effectively, are the cultures of school life itself" [20]. To succeed within the educational system, students must master the cultures of school life. One of the earliest studies of schooling and the educational system is found in Philip W. Jackson's "Life in Classrooms", which was first published in 1968 and has become a landmark in educational research. His studies contribute to an analytic view about the lives of young people in special rooms in special buildings where "classrooms, by and large, are relatively quiet places and it is part of the teacher's job to keep them that way" [16]. Jackson presents the concept of classroom etiquette to cover the required behavior for people in these rooms, who have to adapt to this code of conduct in order to survive. His studies show that the educational system is a specific type of system that requires a high degree of adaptation from students in terms of a specific behavior. For instance, the concept of waiting is of high importance in schools, where the students learn that a classroom is" a place where things often happen not because students want them to, but because it is time for them to occur" [16].

Automatized system adaptation and learning in automatic systems are by no means newcomers in schools and education. They have been players in the field for more than 50 years $[5,21]$, but with the increased investment in digital, educational technology in the past decades, automatized 
system adaptation has grown in terms of learning resources, platforms and various forms of educational technology. Automatized system adaptation is, however, not a paradigm shift in education. Rather, it is what we label as camouflage. The concept of automatized system adaptation is still, in the words of von Foerster, a trivial system because the learner is the one who has to adapt to the system. An automatized system may have various ways for students to enter and pass through, for example, a math assignment, but even though the system is automatized, it is nevertheless based on the premises of the system, and the various paths or learning ways are predetermined within the system itself. It is, in other words, the system that sets the standards and the design for the adaptational work to take place in.

Adaptation seen as recommendation systems set out to empower both students and teachers offers another way of looking at the concept of adaptation. This approach can be described as non-trivial adaptation using von Foerster's terms. Recommendation systems are the opposite of mechanical adaptation; here the starting point is the student, the student's competencies and learning history. Every student has a unique profile, different educational learning paths, and if an educational design includes this as a starting point, the crucial idea is that the system must adapt to the learner and not the other way around. In the next section, we will look further into this approach.

\section{Three Approaches to Solving the Scaling Problem}

In 1984, Benjamin Bloom published the paper "The 2 Sigma Problem", which shows that the instructional design referred to as one-to-one tutoring (one teacher per student) is far more efficient in terms of student learning than group instruction [22]. Bloom concluded that there was a need for research in classroom methods that are as effective as one-to-one tutoring, because this design is "too costly for most societies to bear on a large scale" [22]. Bloom's "2 Sigma Problem" is a scaling problem related to the well-known instructional design model, which visualizes one-to-one tutoring:

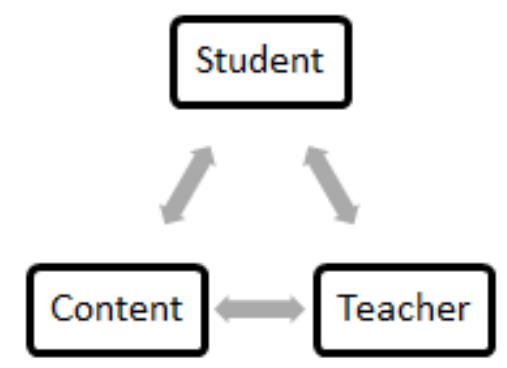

Figure 3. Traditional, instructional design model [23].

The developments of adaptive classroom methods that are able to support differentiation, individualization, and personalization have not been encouraging. In particular, three technology enhanced teaching concepts have been marketed with the promise that they can solve the scaling problem. These concepts try to transform the role of the teacher in the above design model.

- The first concept is adaptive learning systems that replace the teacher with a technical system.

- The second concept seeks to empower the teacher with new digital technologies which will enable the teacher to perform adaptive instructional designs that match each student's personalized learning needs. Learning analytics is the latest technology trend, which aims to support teachers in this teaching task.

- The third concept is the adaptive MOOC (Massive Open Online Courses), which transforms teacher presence into mediated asynchronous teacher telepresence.

For all of the three concepts, implementations that are based on the idea of adaptation as either automatized systems or recommendation systems are possible.

\subsection{Adaptive Learning Systems}

Attempts to individualize instruction with a technical system is an older idea. Frederick Taylor [24] was interested in the idea of a "teaching machine". In 1958, B.F. Skinner introduced the idea of technology mediated programmed learning [25] and in the 70s much research in the field of Computer-Assisted Instruction (CAI) took place. The criticism of this approach, and especially the radical behaviorism developed by Skinner, has been intense in education research for decades. Adaptive learning systems are this century's attempt to develop a "fifth-generation" educational technology [1] adapted to the user's needs.

Most adaptive learning systems consist of three components [6,26]: A content model, a learner model and an instructional design model, which is a strategy for the adaption process.

A content model structures the content of learning objectives, sequences and tasks to be solved [26]. A content model divides the subject into smaller elements, which can be associated with different types of learning resources [27].

An adaptive learning system also contains a model of the learner [28]. The model is based on one or both of the following categories: a) the learner's current knowledge, and b) the learner's learning preferences. The model of the learner must visualize the personalized curriculum a given person should be offered in a concrete course. Most adaptive learning systems therefore identify the learner's existing knowledge and compare the learner's knowledge with the knowledge structure or curriculum for a given subject.

The majority of all commercial adaptive learning systems also try to model the learner's preference for certain types of learning processes. Attempts to categorize the learners in cognitive types or learning styles are very common. In a review of 70 published articles on adaptive learning systems 
[29] $81 \%$ of the participating learning systems used cognitive types or learning styles for modeling learners. Most used were cognitive types based on Kolb [30] and learning styles based on Felder \& Silverman [31] or Dunn and Dunn [32]. Despite the widespread use of models of the learner building on typologies of preferences in terms of learning styles or cognitive types, the same study showed that "findings on concrete learning outcomes were not strong enough" [29].

For this reason, it is important to be critical towards adaptive learning systems that emphasize the identification of specific preferences and hypotheses concerning specific learning styles. Especially because the development of a model of the learner based on hypotheses related to the learner's preferences can develop into what is called "stereotype methods" [33].

The third dimension in an adaptive learning system is the strategy of adaptation. Basically, we can distinguish between two adaptation strategies: Recommendation systems or controlled navigation [44]. In a recommendation system, the technology identifies a range of possibilities, which the system priorities for the learner based on a learner model or on the basis of the learner's performance in the system. The learner is, however, free to choose whether to follow the recommendation or not. By controlled navigation, the system hides the links which are not relevant to the learner, either because they do not match the model of the learner or because they do not match the learner's continuous performance in the system.

An important design discussion is the question of who should have control of the adaption process. Is it the system or the learner $[1,33]$ ? Reviews of research on adaptive learning systems show that this is not always reflected in the design of the adaptive learning system [29, 34]. The problem is that the adaption process may be invisible to the learner, since the rules or algorithms that are used to control the system is not known or understood by the user. The system can collect a large amount of data about the user (big data) through the monitoring of the learner's interactions with the system [1]. This raises a number of ethical questions and dilemmas of privacy and users' control of their own data. Who owns the data produced by an adaptive learning system, and what can and should this data be used for?

\subsection{Learning Analytics}

As we can tell from the above, the solution to solving the scaling problem does not involve replacing the teacher with a technology that performs automatized adaptation on the basis of non-transparent algorithms. In recent years, different types of technologies and a completely different understanding of the teacher's role has gained attention. These concepts shall empower the teacher with technologies that will enable the teacher to perform adaptive instructional designs that match each student's personalized learning needs. Learning analytics is the latest technology trend that aims to support teachers in this teaching task.

Research in learning analytics has been scarce. The first international conference was held in 2011, where learning analytics was defined "as the measurement, collection, analysis and reporting of data and their contexts, for purposes of understanding and optimizing learning and the environments in which it occurs" [35]. Learning analytics uses methods from data mining but with a different purpose. As opposed to data mining, which aims to automate data collection, pattern recognition and perform automated adaptation, learning analytics aims to collect and analyze data in order to support teachers and students in making more informed choices of adaptation strategy $[11,36]$. The differences between the two technologies are summarized by Siemens and Baker [11] in the figure below:

\begin{tabular}{|c|c|c|}
\hline & Learning Analytics & Educational Data Mining \\
\hline Discovery & $\begin{array}{l}\text { Leveraging human judgment is the key; automated } \\
\text { discovery is a tool to accomplish this goal. }\end{array}$ & $\begin{array}{l}\text { Automated discovery is the key; leveraging human } \\
\text { judgment is a tool to accomplish this goal. }\end{array}$ \\
\hline Reduction \& Holism & $\begin{array}{l}\text { Stronger emphasis on understanding systems as } \\
\text { wholes, in their full complexity. }\end{array}$ & $\begin{array}{l}\text { Stronger emphasis on reducing to components and } \\
\text { analyzing individual components and relationships between } \\
\text { them. }\end{array}$ \\
\hline Origins & $\begin{array}{l}\text { Stronger origins in the semantic web, "intelligent } \\
\text { curriculum," outcome prediction and systemic } \\
\text { interventions. }\end{array}$ & $\begin{array}{l}\text { Strong origins in educational software and student } \\
\text { modelling, with a significant community in predicting } \\
\text { course outcomes. }\end{array}$ \\
\hline $\begin{array}{l}\text { Adaptation \& } \\
\text { Personalization }\end{array}$ & $\begin{array}{l}\text { Greater focus on informing and empowering } \\
\text { instructors and learners. }\end{array}$ & $\begin{array}{l}\text { Greater focus on automated adaptation (e.g. by the computer } \\
\text { with no human in the loop). }\end{array}$ \\
\hline $\begin{array}{l}\text { Techniques \& } \\
\text { Methods }\end{array}$ & $\begin{array}{l}\text { Social network analysis, sentiment analysis, influence } \\
\text { analytics, discourse analysis, learner success } \\
\text { prediction, concept analysis, sense-making models. }\end{array}$ & $\begin{array}{l}\text { Classification, clustering, Bayesian modelling, relationship } \\
\text { mining, discovery with models, visualization. }\end{array}$ \\
\hline
\end{tabular}

Figure 4. A brief comparison of the two fields [11] 
As shown in Fig. 4, Siemens and Baker [11] seek to reserve the term learning analytics for technologies that we define as adaptive recommendation systems, which serve the purpose of informing teachers and students about pedagogical practice in order to empower both parties. Educational data mining is, on the other hand, used by Siemens and Baker to describe technologies that rely on automatized adaptation. We acknowledge this essential distinction, but are also aware that learning analytics, in the same way as adaptive learning systems becomes an umbrella term for technologies that can take the form of both recommendation systems and automatized adaptation technologies.

\subsection{Adaptive MOOCs}

The third education concept enhanced by technology that seeks to solve the challenges related to adaptation are adaptive MOOCs. University College Absalon in Denmark has established a research project with the aim of developing a design framework that can guide the development of SPOCs, adapted to experienced teachers' different learning needs and study the factors affecting the actual realization, legitimacy and efficacy of the design.

\section{The Case: An Adaptive SPOC for Teacher Professional Development}

By 2020 , it will be a requirement that Danish primary school teachers have a bachelor degree in the subjects they teach. More than 10,000 teachers, who have for many years taught a course without being formally qualified, need professional development and therefore municipalities ask for new training concepts. There is a need for educational concepts that are flexible in relation to teachers' work situations and are based on the fact that the teachers already have acquired a number of professional skills. A number of municipalities (the customers) and University College Absalon (UCA, the provider) are in the process of examining whether the training format SPOC (Small Private Online Course) can solve this training task. As part of this process,
UCA has established a research project with the aim of developing a design framework that can guide the development of SPOCs in our institution.

Methods. The project is methodologically inspired by Design-Based Research (DBR) [37, 38]. Informed by previous research in MOOCs, adaptive learning systems and learning Analytics, the design framework has been developed through iterative design experiments [39]. Several prototypes have been evaluated and redesigned. We have analyzed interviews with participants and teachers and made observations of the participants' interactions with each other and with the technology (the learning platform Moodle). Through these design experiments, it has been possible to develop a design framework consisting of a set of pedagogical design principles.

Design Criteria for an Adaptive SPOC. Based on prior research, we have defined a set of design criteria for the development of an adaptive learning design for SPOCs:

- Modeling of the learner must be based on documented effects.

- The development of an adaptive learning design must be based on a precautionary principle (ethical code), which means that we do not use stereotypical methods for modelling the learner.

- Modeling should (only) visualize 1) the learner's professional skills and 2) experience and skills to learn in a given training format, e.g. MOOCs/SPOCs.

- Adaptation performed by a technical system based on non-transparent algorithms cannot stand alone.

- Adaptation must be a dialogue (negotiation) between the learner and a teacher on the basis of one or more technically generated information.

- The adaption strategy should be recommendations and the adaptation process must be transparent and controlled by the learner.

The Design Framework. Based on a series of design workshops and three iterative design experiments, we have developed a design framework for the design of adaptive learning environments in formal education based on SPOCs: 


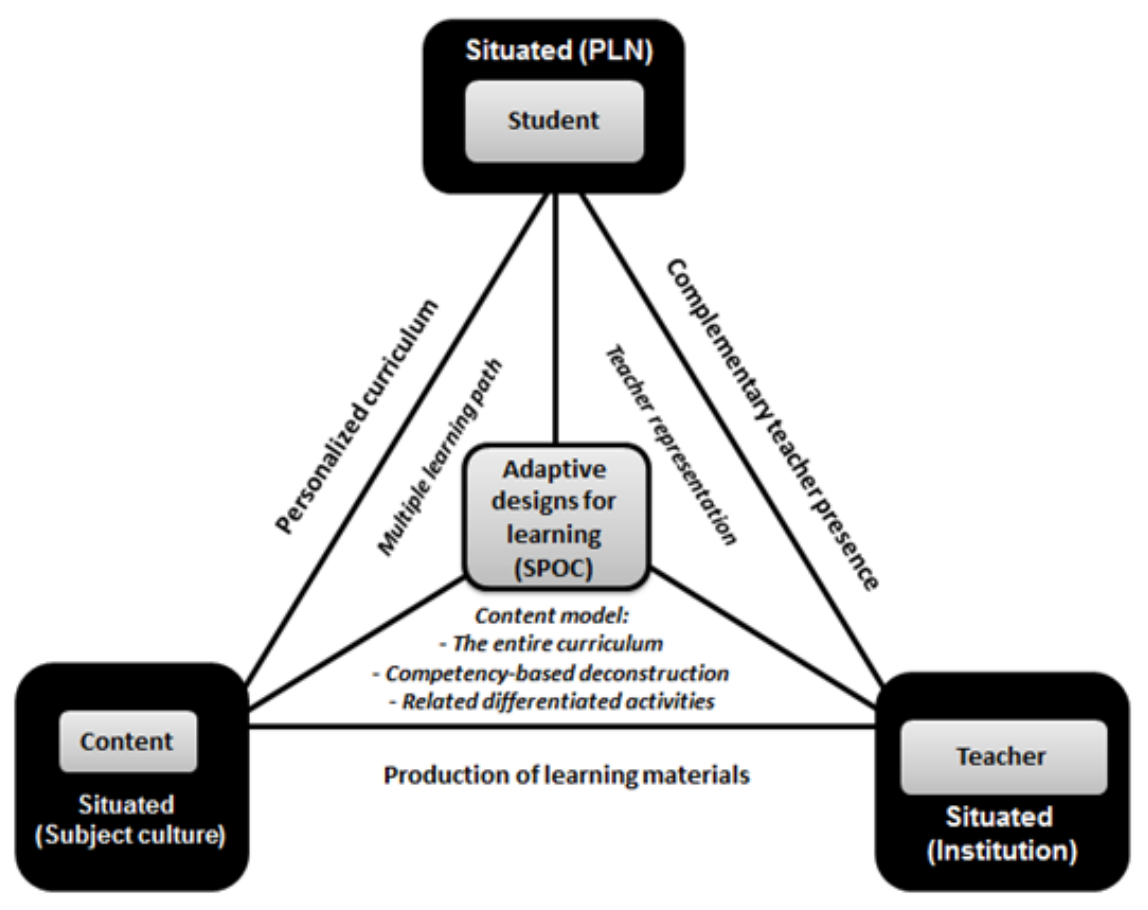

Figure 5. Design framework for an adaptive hybrid SPOC [inspired by 45].

The design framework visualizes three design levels:

Setting. The design framework is based on a well-known design model that frames the design as a setting for formal training with a student, content and a teacher. However, we are following the widespread criticism of this model and situate the three elements of the model in the context they are part of [23]. The student is part of a personal learning network (PLN). The content is part of a broader academic culture and its interpretation of the subject. The teacher is situated in an educational institution and more widely in an educational system. The development of a specific adaptive learning design must be based on the framing and the concrete anchorage of the three elements in their specific contexts.

Relationships. The framework visualizes the characteristics of the relationship between the designed elements described above. The relationship between the student and the content is characterized by a personalized curriculum. Each student has his or her own unique curriculum. The relationship between a student and the teacher is also characterized by complementarity. In traditional teaching concepts, the relation between a student and the teacher is the core of the instructional design and teacher presence is the starting point for concrete designs for learning. However, this is not possible in an instructional design where all participants have their own personalized curriculum. In a group of students who each have their own curriculum, it is not possible to realize a multiple relationship: a student - a content - a teacher. The relationship between student and teacher must be complementary if you want to support that all participants have a personalized curriculum. Finally, the relationship between the teacher and the subject also has a characteristic feature that is far from usual perceptions about being a teacher. The traditional role of the teacher is the lecturer who interprets a subject and mediates the relationship between the student and the subject in a face-to-face setting. The project shows that the relationship between the teacher and the subject must be transformed from a teacher role to an author role. The teacher is rather a designer, an author and a producer of a number of learning resources, a role that also entails that the teacher is part of a larger production team.

Principles. Level 3 in the model visualizes the design principles. These principles relate to each of the three characteristics described above.

Personalized Curriculum: Multiple Learning Paths. The design must be able to identify the participant's current skills - visualized in a competency profile. The design must be able to visualize a competence-gap in terms of a personalized curriculum. Finally, the design must be able to recommend a learning path that adaptively matches the learner's personalized curriculum. We will refer to the principle of potentially multiple learning pathways, and thus the possibility of recommending an adaptive personalized learning path for each student, as the design potential or affordance of the design.

Production of Learning Resources: The Content Model. In order to realize the principle of multiple pathways of learning, the educational institution in advance has to produce a content model, that covers the entire curriculum of the subject, includes deconstruction of the subject to competency units and guides the production of learning resources and forms of participation, which without progression are linked to each unit of competence. This design principle can be described as a constraint for adaptive learning designs. The design framework includes no constraints regarding the 
choice of types of activity associated with specific stereotypes, learning styles, etc. The framework thus encourages the development of a number of different types of activity associated with each competency.

Complementary Teacher Presence: Representation of the Teacher. The final design principle is a key constraint for the design of MOOCs in general and thus also for an adaptive SPOC. Since the teacher cannot be present in a potentially multiple number of learning pathways, the teacher must be represented in the design. The teacher must be mediated in a form that minimizes the disadvantage of a learning design where the teacher cannot be physically present. The principle of complementary teacher presence can be formulated as a scale, and an educational institution must in each case decide the extent to which it will complement the asynchronous teacher presence with synchronous presence forms either online or on campus. Besides these asynchronous and synchronous presence forms, Christiansen \& Rosenlund have put forward the concept of Presence-Absence as a certain form of presence that is characterized as: "The psychological state or subjective perception in which a part of or all of a student's perception fails to acknowledge the role of technology in his or her current experience of a learning situation involving a teacher represented digitally in such a way that he or she is recognized and acknowledged as a teacher" [40]. This can be done in numerous ways and one of them could be through meaningful videos where the teachers use e.g. humor and present some of their own thoughts on a subject or a discussion. It is crucial that students get the feeling that real human beings are behind the learning environment in which they participate.

Evaluation of the Design Framework. The evaluation of the developed design principles follows methods and guidelines from Design-Based Research [41, 42]. In our evaluation, we distinguish between the design principles outlined in the framework above, and specific designs for learning developed by individual teachers/authors in a given educational institutionalized context. In a DBR project, there is no straight line from the developed theory (design principles) and the actual design solution. "Design principles are not intended as recipes for success, but to help others select and apply the most appropriate substantive and procedural knowledge for specific design and development tasks in their own settings" [42]. Specific learning designs can therefore easily develop into mutations (legitimate or lethal), which research should subsequently study in order to revise the developed theory [43].

The designs developed by the teachers/authors, have been evaluated according to feasibility, legitimacy and efficacy [42]. The degree of feasibility, legitimacy and efficacy affects the intended design that an educational institution produces and offers to its customers. However, the intended design is not the same as the implemented and the attained designs. The intended design is what the design is set out to do. The implemented design is how it is actually used in practice by teachers and students, and the attained design is the specific outcome of the design - in our case the learning outcome [42]. An evaluation design must test both the intended, the implemented and the attained designs.

The evaluation was conducted using alpha testing and beta testing [42]. Our alpha trials have been controlled by the research team with maximum support for teachers and students. The aim was to test the feasibility of the design in our institution and explore the teachers' and students' assumptions about viability and impact on learning outcome. In our beta test, we have tested the SPOC in a real life context but still with some support. The goal has been to explore conflicts between the intended design and its implementation according to institutional feasibility and viability, map out fostering and hindering conditions for implementation and measure the initial impact on learning outcome.

\section{Results}

\subsection{The Choice of Intended Design}

By now, UCA has produced SPOCs in seven different subjects and has decided to produce SPOCs for all subjects taught in the Danish primary school by 2018. The SPOCs are for in-service training for experienced school teachers without formal qualifications, in the following referred to as "the students".

The first step before entering the course is self-assessment. On a scale from 1- 5 the student assesses his or her qualifications in relation to the objectives of the course, which are formulated in terms of competencies. The self-assessment is conducted with a tool developed within the project. On the basis of the student's input, the tool generates and visualizes a competence-profile illustrating the percentage of the curriculum, the student must study. This happens automatically on the basis of a simple algorithm that collects all of the competency outcomes rated 3 or less by the student. The developed tool also serves as a recommendation and navigation system that generates an adaptive match between the competence-gap and selected study themes. The SPOC platform for each of the 7 subjects is designed so adaptation is possible, no matter what skills the student needs to pass the exam. The curriculum in a subject is divided into a number of themes that are organized so they can be accessed without progression. Each theme is assigned a set of competencies that the students can acquire through study work, by accessing video resources, texts, exercises, quizzes and participation in peer-to-peer response, collaboration etc. Again, the system performs simple automatized adaptation to match the competency profile with the relevant study themes, but the recommendation system is supplemented by a guidance session with a teacher, because UCA wishes to supplement the technical recommendation system with a dialogue with each student. Through a $90 \mathrm{~min}$. dialogue with a teacher, the self-evaluation is reviewed, and the teacher provides additional guidance on the selection of adaptive 
themes and navigation in the SPOC platform. The evaluation shows that there is a high degree of fidelity between the design principles and the intended design of the 7 SPOCs that UCA has produced to this day.

\subsection{The Implemented and Attained Designs}

The evaluation of the implemented design shows that there are four different clusters of mechanisms that have significance for the attained design in different local contexts:

- The participants' perception of relevance and usefulness of the intended learning design including the recommendation system.

- UCA's introduction of the intended design to the students.

- The students' study conditions granted by their employer.

- The students' academic qualifications.

Implementation of the Recommendation System. In this paper, we have focused on the first clusters of mechanism and the implementation and effect of the recommendation system in particular. The evaluation shows that both the students and teachers find the recommendation system and the additional guidance session with a teacher useful at the beginning of the course, but subsequently the recommendation loses its value. The evaluation shows that the "recommendation system" appreciated the most by the students is situated practice within the SPOC, which means that the students only start to believe that they are competent once they have worked with the competency outcomes and the related resources in the SPOC. Furthermore, the evaluation shows that the most important recommendation system offered by this educational concept is the open access for all students which allows them to work with all competencies within a subject when they wish to do so. The opportunity to work with the resources for a full education programme is valued the most by the students, and in interviews they even express the wish to have continuous access to an archived version of the SPOC once they have completed the course.

The most crucial finding of the project in relation to working with recommendation systems is the fact that simply implementing a recommendation system at the start of a course is insufficient; a recommendation system can only support the development of adaptive and personalized learning paths for each student if the system is employed throughout the course. Moreover, this will only be possible if the full education program is temporarily and spatially transparent and available in relation to each student's wishes for their course of study. This presupposes a shift in our understanding of education from being a relation between a class of students, a teacher and the content to being a learning path within a learning environment where everything is available at all times and where a unique path is recommended for each student. Only then will we reach a changing paradigm, where the education system becomes a recommendation system rather than a matter of schooling.

\section{Further Research}

The discussions in this paper raise a number of questions in relation to educational adaptation, and further research within the field is required.

Firstly, the development of recommendation systems in educational design solutions should be followed and examined closely. The further development of recommendation systems should be carried out via experiments, iterations and guidance from researchers as well as teachers. Secondly, the emergence of various types of sub-MOOCs is likely to result in different types of adaptation forms and, presumably, adaptation in such MOOCs will play different roles in different contexts. Furthermore, the continuing development of sub-MOOCs calls for further research into what is required from teachers and students who interact with different types of recommendation systems [40].

The shift in education from educational systems requiring student adaptation to educational recommendation systems adapting to students' individual needs requires that new concepts for discussing education and adaptation are developed. Moreover, future research should explore how paradigm shifts occur within an educational setting and how such ground-breaking changes can be supported on an organizational level. Finally, we need to ask ourselves the question: What will be the consequences of educational systems mutating into a multitude of recommendation systems, and how can we meet the challenges and demands that will follow?

\section{REFERENCES}

[1] Siemens, G., Gasevic, D. \& Dawson, J.: Preparing for the Digital University (2015). A Review of the Current State of Distance, Blended and Online Learning. Athabaska University. Retrieved 25.07.17 from: http://linkresearchlab.org/PreparingDigitalUniversity.pdf.

[2] Shute, V. J. \& Zapata-Rivera, D. (2008). Adaptive Technologies. In: Spector, J. M., Merrill, M. D., Merrienboer, J. van \& Driscoll, M. P. (eds.) Handbook of Research on Educational Communications and Technology. Springer-Verlag, New York, pp. 277-294.

[3] Darwin, C. (1859). On the Origin of Species by Means of Natural Selection, or, the Preservation of Favoured Races in the Struggle for Life. John Murray, London.

[4] Spencer, H (1855). The Principles of Psychology. Longman, Brown, Green \& Longmans, London.

[5] Cronbach, L. J. (1957). The two Disciplines of Scientific Psychology. American Psychologist. 12(11), pp. 617-684. 
[6] Oxman, S. \& Wong, W. (2014). White Paper: Adaptive Learning Systems. Integrated Education Solutions.

[7] Baggaley, J. (2014). MOOC postscript, Distance Education, $35: 1$, pp. 126-132.

[8] Horizon Shortlist Report 2013, Higher Education Edition (2013). Retrieved 04.04.2016 from http://www.nmc.org/pdf/2013-horizon-higher-ed-shortlist.pdf

[9] Evans, S. \& Maryck, G. (2015). How MOOC Instructors View the Pedagogy and Purposes of Massive Online Course. Distance Education, issue 3, pp. 295-311.

[10] Siemens, G.: Connectivism: Learning as Network Creation. Elearnspace (2005). Retrieved 22.07.2017 from http://www.elearnspace.org/Articles/networks.htm

[11] Siemens, J. \& Baker, J. (2012). Learning Analytics and Educational Data Mining: Towards Communication and Collaboration. Learning Analytics and Knowledge. Proceedings of the $2^{\text {nd }}$ International Conference on Learning Analytics and Knowledge, pp. 252-254.

[12] Bayne, S. \& Ross, J. (2014). The Pedagogy of the Massive Open Online Course: The UK View. The Higher Education Academy, University of Edinburgh (2014). Retrieved 03.07.17 from:

https://www.heacademy.ac.uk/sites/default/files/hea_edinbur gh_mooc_web_240314_1.pdf

[13] Blume, R. G. (2012). Trivialisierung und/oder Empowerment? Lecture at Ludwig Boltzman Institut Health Promotion Research, Vienna, June 2.12.2012.

[14] Foerster, Heinz von (1993). : Mit den Augen des Anderen. In: Schmidt, S. J. (ed.) Wissen und Gewissen. Suhrkamp Verlag-

[15] Bourdieu, P. \& Passeron, J. C. (1990). The Reproduction in Education. Sage Publications.

[16] Jackson, P. W. (1968). Life in Classrooms. Holt, Rinehart and Winston Inc., Chicago.

[17] Lave, J. \& Wenger, E. (1991). Situated Learning. Legitimate Peripheral Participation. University Cambridge Press.

[18] Kincheloe, J. \& Steinberg. S (eds.) (1998). Students as Researchers - Creating Classrooms that Matter. Routledge.

[19] Trilling, B. \& Fadel, C. (200). $21^{\text {st }}$ Century Skills - Learning for Life in Our Times. The Partnership for $21^{\text {st }}$ Century Learning Skills.

[20] Brown, J. S., Collins, A. \& Duguid, P. (1989). Situated Cognition and the Culture of Learning. In: Educational Researcher, Vol. 18, No. 1, pp. 32-42.

[21] Tsypkin, Y. Z. (1971). Adaptation and Learning in Automatic Systems. Academic Press.

[22] Bloom, B. S. (1984). The 2 Sigma Problem: The Search for Methods of Group Instruction as Effective as One-to-One Tutoring. In: Educational Researcher, vol. 13, no. 6, pp. 4-16.

[23] Garisson, D. R. \& Anderson, T. (2011). E-learning in the 21st Century: A Framework for Research and Practice. Routledge/Falmer, London.

[24] Taylor, F. (1911). The Principles of Scientific Management. Harper \& Brothers, New York.
[25] Skinner, B. E. (1958). Teaching Machines. Science, 128, pp. 969-977.

[26] Natriello, G. (2011): Adaptive Educational Technologies and Educational Research: Opportunities, Analyses, and Infrastructure Needs. Background Paper Prepared for the National Academy of Education. Retrieved 25.03.16 from: http://www.naeducation.org/cs/groups/naedsite/documents/w ebpage/naed_080845.pdf

[27] Thalmann, S. (2014). Adaption Criteria for the Personalized Delivery of Learning Materials: A Multi-stage Empirical Investigation. In: Australasians Journal of Educational Technology, 30(1).

[28] Wenger, E. (1987). Artificial Intelligence and Tutoring Systems. Morgan Kaufmann Publishers Co., Los Altos, CA.

[29] Akbulut, Y. \& Cardak, C. S. (2012). Adaptive Educational Hypermedia Accommodating Learning Styles: A Content Analysis of Publications from 2000 to 2011. In: Computers \& Education. vol. 58, pp. 835-842.

[30] Kolb, D. (1984). Experiential Learning. Prentice Hall.

[31] Felder, R. M., \& Silverman, L. K. (1988). Learning and Teaching Styles in Engineering Education. Engineering Education, 78(7), pp. 674-681.

[32] Dunn, R. \& Dunn, K. (1974). Learning Style as a Criterion for Placement in Alternative Programs. Phi Delta Kappan 56(4), pp. 275-278.

[33] Shute, V. J. \& Zapata-Rivera, D. (2012). Adaptive Educational Systems. In Durlach, P. \& Lesgold, A. Adaptive Technologies for Training and Education. Cambridge University Press, pp. 7- 27.

[34] Ford, K.: Adaptive Learning Bibliography (1993). Retrieved 03.07.17 from:

http://www.umuc.edu/innovatelearning/upload/adaptive-learn ing-an-annotated-bibliography.pdf

[35] Pardo, A. \& Siemens, G. (2014). Ethical and Privacy Principles for Learning Analytics. British Journal of Educational Technology. Vol. 45 No 3, pp. 438-450.

[36] Long, P. \& Siemens, G. (2011). Penetrating the Fog: Analytics in Learning and Education. Educause Review, 48, 5, pp. 3140 .

[37] Brown, A. L. (1992). Design Experiments: Theoretical and Methodological Challenges in Creating Complex Interventions in Classroom Settings. Journal of the Learning Sciences, 2(22), pp. 141-178.

[38] Collins, A. (1992). Towards a Design Science of Education. In: E. Scanlon \& T. O'Shea (eds.), New Directions in Educational Technology, pp. 15-22. Springer, Berlin.

[39] diSessa, A. A., \& Cobb, P. (2004). Ontological Innovation and the Role of Theory in Design Experiments. Journal of the Learning Sciences, 13(1), pp 77-103.

[40] Christiansen, R. B. \& Rosenlund, L. T. (2016). Is there Anybody in here - Present-Absence, Positions and Relations in MOOCs. Designs for Learning.

[41] Akker, J. V. D., Gravemeijer, K., Mckenney, S. \& Nieveen, N. (eds.) (2006). Educational Design Research. Routledge, London \& New York. 
[42] McKenney, S. \& Reeves, T.C. (2012). Conducting Educational Design Research. London \& New York, Routledge.

[43] Hung, D. et al. (2010). Extending and Scaling Technology-based Innovations through Research. In. OECD: Inspired by Technology, Driven by Pedagogy - A Systemic Approach to Technology-based School Innovations.

[44] Khribi, M. K., Jemni, M. \& Nasraoui, O. (2015) Recommendation Systems for Personalized
Technology-enhanced Learning. In: Kinshuk, R. H. (ed.) Ubiquitous Learning Environments and Technologies. Springer, Berlin.

[45] Gynther, K. (2015. Design Framework for an Adaptive, Hybrid MOOC: Personalized Curriculum in Teacher Professional Development. In: Jefferies, A. \& Cubric, M. (eds.) Proceedings of the $14^{\text {th }}$ European Conference on e-learning, University of Hertfordshire Hatfield, UK 29-30 October 2015, pp. 255-264. 